Raymond J. Grinnell, Department of Mathematics and Computer Science, Mount Allison University, 67 York Street, Sackville, NB E4L 1B3, Canada. e-mail: rgrinnell@mta.ca

\title{
FUNCTIONS PRESERVING SEQUENCE SPACES
}

\begin{abstract}
Let $A$ and $B$ be sets of real sequences. Let $F(A, B)$ denote the set of functions $f: \mathbb{R} \rightarrow \mathbb{R}$ that preserve $A$ and $B$ in the sense that $\left(f\left(a_{n}\right)\right) \in B$ for all sequences $\left(a_{n}\right) \in A$. These functions are generalizations of convergence preserving functions first introduced by Rado. We establish identities and inclusions for $F(A, B)$ when $A$ and $B$ are $l^{p}$-spaces and other well-known sequence spaces. We also characterize $F(A, B)$ in terms of elementary classes of functions. Our characterizations are motivated by the work of Borsík, Červeňanský and Šalát.
\end{abstract}

\section{Introduction and Notation}

A function $f: \mathbb{R} \rightarrow \mathbb{R}$ is said to be convergence preserving provided that $\sum_{n=1}^{\infty} f\left(a_{n}\right)$ converges whenever the real series $\sum_{n=1}^{\infty} a_{n}$ converges. These functions are introduced by Rado in [2] where it is shown that $f$ is convergence preserving if and only if $f$ is linear near the origin. This characterization is first established by Rado in the setting of Banach spaces. The real-variable result is then derived as a special case. This theorem is also obtained in [6] via purely real-variable arguments and an even simpler proof is found in [4]. Generalizations of convergence preserving functions are investigated by Borsík, Červeňanský and Šalát in [1]. Their study entails the $f$-transform concept. Given a function $f: \mathbb{R} \rightarrow \mathbb{R}$ the $f$-transform of the series $\sum_{n=1}^{\infty} a_{n}$ is the series $\sum_{n=1}^{\infty} f\left(a_{n}\right)$. Three new classes of functions are defined in [1], in addition to convergence preserving functions. These classes are the four combinations obtained when the domain and range of the $f$-transform are chosen pairwise from the set of convergent and absolutely convergent series.

In this paper we investigate functions for which the domain and range of the $f$-transform are extended to sequence spaces. Specifically, if $A$ and

Key Words: Function preserving convergence, infinite series, sequence space

Mathematical Reviews subject classification: 26A99, 40A05, 46A45

Received by the editors August 4, 1998 
$B$ are sets of real sequences we study the class of functions $f$ such that the transformed sequence $\left(f\left(a_{n}\right)\right)$ belongs to $B$ for each sequence $\left(a_{n}\right)$ in $A$. A number of our results extend those in [1] and [2]. Some these were announced at the Summer Symposium in Real Analysis, XXII, at Santa Barbara.

Let $\left(a_{n}\right)$ denote the sequence whose $n^{\text {th }}$-term is the real number $a_{n}$ and let $(0)$ denote the zero sequence. We work with the following sequence spaces.

$$
\begin{aligned}
& \omega=\left\{\left(a_{n}\right): a_{n} \in \mathbb{R}\right\} . \\
& c_{0}=\left\{\left(a_{n}\right): a_{n} \rightarrow 0 \text { as } n \rightarrow \infty\right\} . \\
& c_{00}=\left\{\left(a_{n}\right): a_{n} \neq 0 \text { for finitely many } n\right\} . \\
& c s=\left\{\left(a_{n}\right): \sum_{n=1}^{\infty} a_{n} \text { converges }\right\} . \\
& l^{p}=\left\{\left(a_{n}\right): \sum_{n=1}^{\infty}\left|a_{n}\right|^{p} \text { converges }\right\}, p \in(0, \infty) . \\
& l^{\infty}=\left\{\left(a_{n}\right): \sup \left\{\left|a_{n}\right|\right\}<\infty\right\} .
\end{aligned}
$$

Let $\mathcal{I}=\bigcap_{0<p<\infty} l^{p}, \quad \mathcal{U}=\bigcup_{0<p<\infty} l^{p}$ and $\mathcal{S}=\left\{c_{0}, c_{00}, c s, l^{p}(p \in(0, \infty]), \mathcal{I}, \mathcal{U}\right\}$.

The symbol $\subset$ will denote proper containment and $\subseteq$ will denote containment which may entail equality. Some of our results depend on inclusions for the spaces above. An important chain of inclusions is that

$$
c_{00} \subset \mathcal{I} \subset l^{r_{1}} \subset \bigcap_{r_{1}<s<r_{2}} l^{s} \subset \bigcup_{r_{1}<t<r_{2}} l^{t} \subset l^{r_{2}} \subset \mathcal{U} \subset c_{0} \subset l^{\infty}
$$

where $0<r_{1}<r_{2}<\infty$. Furthermore, if $0<p \leq 1$ then

$$
l^{p} \subset c s \subset c_{0} \text { but } c s \nsubseteq \mathcal{U} \text {. }
$$

Definition 1.1. For $A, B \subseteq \omega$ let $F(A, B)=\left\{f: \mathbb{R} \rightarrow \mathbb{R}:\left(f\left(a_{n}\right)\right) \in B\right.$ for all $\left.\left(a_{n}\right) \in A\right\}$.

The spaces $F(A, B)$ entail those in [1] when $A, B \in\left\{c s, l^{1}\right\}$ :

$$
\begin{aligned}
F^{(c p)} & =F(c s, c s), & F^{(a c p)} & =F\left(l^{1}, l^{1}\right), \\
F^{(a c, c)} & =F\left(l^{1}, c s\right), & F^{(c, a c)} & =F\left(c s, l^{1}\right) .
\end{aligned}
$$




\section{Basic Identities and Inclusions}

We are mostly interested in studying the spaces $F(A, B)$ where $A, B \in \mathcal{S}$. In all of these cases $F(A, B) \neq \phi$ since each such space contains the zero function, $z(x)=0, x \in \mathbb{R}$. There are certain choices of $A, B \in \mathcal{S}$ for which $F(A, B)=\{z\}$. These will appear in the next section. The first proposition is a useful consequence of Definition 1.1.

Proposition 2.1. Let $A_{1}, A_{2}, B_{1}, B_{2} \subseteq \omega$.

(i) If $B_{1} \subseteq B_{2}$ then $F\left(A_{1}, B_{1}\right) \subseteq F\left(A_{1}, B_{2}\right)$;

(ii) If $A_{1} \subseteq A_{2}$ then $F\left(A_{2}, B_{1}\right) \subseteq F\left(A_{1}, B_{1}\right)$.

The notation in (3) and [1, Remark 2.11] imply that

$$
F\left(c s, l^{1}\right) \subset F(c s, c s) \subset F\left(l^{1}, l^{1}\right) .
$$

Moreover, [1, Theorem 2.6] contains the surprising identity

$$
F\left(l^{1}, l^{1}\right)=F\left(l^{1}, c s\right) .
$$

It will follow from several characterization theorems in the next section that for certain $A_{1}, B_{1}, B_{2} \in \mathcal{S}$ where $B_{1} \subset B_{2}$, we have $F\left(A_{1}, B_{1}\right)=F\left(A_{1}, B_{2}\right)$. Here is an important example of this. Let $q \in(0, \infty)$. Assume $f \in F\left(l^{\infty}, l^{q}\right)$ and $x \in \mathbb{R}$. The constant sequence $\left(a_{n}\right)$, where $a_{n}=x$, is an element of $l^{\infty}$. It follows that $\left(f\left(a_{n}\right)\right) \in l^{q}$ which implies $f(x)=0$. This proves that for each $q \in(0, \infty)$,

$$
F\left(l^{\infty}, l^{q}\right)=\{z\} .
$$

Proposition 2.2. Let $p, r_{1} \in(0, \infty]$ and $r_{2} \in[0, \infty)$. Then we have the following:

(i) $\bigcup_{0<s<r_{1}} F\left(l^{p}, l^{s}\right)=F\left(l^{p}, \bigcup_{0<s<r_{1}} l^{s}\right)$;

(ii) $\bigcap_{s>r_{2}} F\left(l^{p}, l^{s}\right)=F\left(l^{p}, \bigcap_{s>r_{2}} l^{s}\right)$;

(iii) $F\left(l^{p}, l^{r_{1}}\right) \subseteq F\left(l^{p}, l^{r_{2}}\right)$ whenever $r_{1}<r_{2}$ and the inclusion is proper if $p<\infty$.

Proof. (i) If $p=\infty$ then the proof of (5) shows that $F\left(l^{\infty}, \bigcup_{0<s<r_{1}} l^{s}\right)=\{z\}$ so we get equality in this case. Assume $p<\infty$. It is easy to verify $(\subseteq)$ using Definition 1.1. We prove $(\supseteq)$ by contradiction. Suppose first that $r_{1}<\infty$ and 
$f \in F\left(l^{p}, \bigcup_{0<s<r_{1}} l^{s}\right)$ but $f \notin \bigcup_{0<s<r_{1}} F\left(l^{p}, l^{s}\right)$. Choose a natural number $k_{0}>1 / r_{1}$ and let $s_{k}=r_{1}-\left(k+k_{0}\right)^{-1}$ for $k \in \mathbf{N}$. There exists a sequence $\left(a_{k, n}\right) \in l^{p}$ such that $\left(f\left(a_{k, n}\right)\right) \notin l^{s_{k}}$ and $\sum_{n=1}^{\infty}\left|a_{k, n}\right|^{p} \leq 1 / 2^{k}$. Let $\left(b_{m}\right)$ be the usual diagonal enumeration of the set $\left\{a_{k, n}: k, n \in \mathbf{N}\right\}$ where $m$ is defined by the pairing function $m=\frac{1}{2}(k+n)(k+n-1)-(k-1)$. The sequence $\left(b_{m}\right) \in l^{p}$ since for each $N \in \mathbb{N}$,

$$
\sum_{m=1}^{N}\left|b_{m}\right|^{p} \leq \sum_{k=1}^{N} \sum_{n=1}^{N}\left|a_{k, n}\right|^{p} \leq \sum_{k=1}^{N} 2^{-k}<1 .
$$

However, the transformed sequence $\left(f\left(b_{m}\right)\right) \notin l^{s_{k}}$ for any $k$ since

$$
\sum_{m=1}^{N(N+1) / 2}\left|f\left(b_{m}\right)\right|^{s_{k}} \geq \sum_{n=1}^{N}\left|f\left(a_{k, n}\right)\right|^{s_{k}} \rightarrow \infty
$$

as $N \rightarrow \infty$. This shows $\left(f\left(b_{m}\right)\right) \notin F\left(l^{p}, \bigcup_{0<s<r_{1}} l^{s}\right)$ which is a contradiction. That establishes $(\supseteq)$ when $r_{1}<\infty$. For $(\supseteq)$ in the case where $r_{1}=\infty$, the proof is identical except that we let $s_{k}=k$.

(ii) If $p=\infty$ then equality follows from (5); if $p<\infty$ then equality is easily verified using Definition 1.1.

(iii) If $p=\infty$ then equality follows once more from (5). If $p<\infty$ then $(\subseteq)$ in (iii) is easily verified. The proper containment in (iii) is established by example. Let $f(x)=|x|^{p / r_{2}}$ for $x \in \mathbb{R}$. For each $\left(a_{n}\right) \in l^{p}$,

$$
\sum_{n=1}^{\infty}\left|f\left(a_{n}\right)\right|^{r_{2}}=\sum_{n=1}^{\infty}\left|a_{n}\right|^{p}<\infty
$$

and therefore $\left(f\left(a_{n}\right)\right) \in l^{r_{2}}$. This shows $f \in F\left(l^{p}, l^{r_{2}}\right)$. Choose a sequence $\left(b_{n}\right) \in l^{p}$ such that $\left(b_{n}\right) \notin l^{p_{1}}$ for any $p_{1}<p$. Then

$$
\sum_{n=1}^{\infty}\left|f\left(b_{n}\right)\right|^{r_{1}}=\sum_{n=1}^{\infty}\left|b_{n}\right|^{\left(r_{1} p\right) / r_{2}}=\infty
$$

since $\left(r_{1} p\right) / r_{2}<p$. This proves $f \notin F\left(l^{p}, l^{r_{1}}\right)$ which gives proper containment.

The next result also gives inclusions for $F(A, B)$ when $A$ or $B$ is an $l^{p_{-}}$ space or union or intersection of $l^{p}$-spaces. However, unlike Proposition 2.2, the inclusions are proper. 
Proposition 2.3. Let $r_{1} \in(0, \infty], q \in(0, \infty)$ and $r_{2} \in[0, \infty)$. Then we have the following:

(i) $F\left(\bigcup_{0<p<r_{1}} l^{p}, l^{q}\right) \subset \bigcup_{0<p<r_{1}} F\left(l^{p}, l^{q}\right)$;

(ii) $\bigcap_{p>r_{2}} F\left(l^{p}, l^{q}\right) \subset F\left(\bigcap_{p>r_{2}} l^{p}, l^{q}\right)$;

(iii) $F\left(l^{r_{2}}, l^{q}\right) \subset F\left(l^{r_{1}}, l^{q}\right)$ whenever $r_{1}<r_{2}$.

Proof. In each assertion $(\subseteq)$ is easily verified using Definition 1.1 and (5). We demonstrate proper inclusion with examples.

(i) Fix $p_{1} \in\left(0, r_{1}\right)$ and let $f(x)=|x|^{p_{1} / q}$. A similar calculation to that in the proof of Proposition 2.2(iii) shows that $f \in F\left(l^{p_{1}}, l^{q}\right)$ and consequently $f \in \bigcup_{0<p<r_{1}} F\left(l^{p}, l^{q}\right)$. Let $p_{2} \in\left(p_{1}, r_{1}\right)$ and choose a sequence $\left(b_{n}\right) \in l^{p_{2}}$ such that $\left(b_{n}\right) \notin l^{s}$ for any $s<p_{2}$. Clearly $\left(b_{n}\right) \in \bigcup_{0<p<r_{1}} l^{p}$. However,

$$
\sum_{n=1}^{\infty}\left|f\left(b_{n}\right)\right|^{q}=\sum_{n=1}^{\infty}\left|b_{n}\right|^{p_{1}}=\infty
$$

since $p_{1}<p_{2}$. This proves $f \notin F\left(\bigcup_{0<p<r_{1}} l^{p}, l^{q}\right)$.

(ii) We argue similarly as above. Fix $p_{1} \in\left(r_{2}, \infty\right)$ and again let $f(x)=$ $|x|^{p_{1} / q}$. Then $f \in F\left(l^{p_{1}}, l^{q}\right)$ and the transformed sequence $\left(f\left(a_{n}\right)\right) \in l^{q}$ whenever $\left(a_{n}\right) \in \bigcap_{p>r_{2}} l^{p}$. This proves $f \in F\left(\bigcap_{p>r_{2}} l^{p}, l^{q}\right)$. Let $p_{2} \in\left(p_{1}, \infty\right)$ and choose a sequence $\left(b_{n}\right) \in l^{p_{2}}$ such that $\left(b_{n}\right) \notin l^{s}$ for any $s<p_{2}$. A small calculation shows $\left(f\left(b_{n}\right)\right) \notin l^{q}$ which implies $f \notin \bigcap_{p>r_{2}} F\left(l^{p}, l^{q}\right)$.

(iii) Let $r \in\left(r_{1}, r_{2}\right)$ and let $f(x)=|x|^{r / q}$ to get the proper inclusion.

Theorem 3.4 in the next section shows that the proper inclusions in Proposition 2.3 are replaced with equality when $q=\infty$. Linear and algebraic structure properties for the spaces $F(A, B)$ are established in the following result which also extends [1, Theorem 2.8 and Remark 2.9] and [5, p. 542].

Proposition 2.4. Let $A, B \subseteq \omega$. 
(i) If $A$ and $B$ are linear sequence spaces then $F(A, B)$ is a linear space under function addition and scalar multiplication;

(ii) Let $A, B \in \mathcal{S}$. The space $F(A, B)$ is closed under multiplication of functions if and only if $B \in \mathcal{S} \backslash\{c s\}$ or $A=c_{00}$;

(iii) The space $F(A, B)$ is closed under function composition whenever $B \subseteq$ $A$.

Proof. (i) The proof is straightforward.

(ii) Sufficiency is obvious if $A=c_{00}$. If $B \in \mathcal{S} \backslash\{c s\}$ and $A \in \mathcal{S}$, then $B \subseteq l^{\infty}$ by (1), hence sufficiency in (ii) follows easily. We prove necessity in (ii) with a counterexample. Let $a_{n}=n^{-n}$ and $E_{n}=\left((n+1)^{-(n+1)}, n^{-n}\right]$ for $n \in \mathbb{N}$. Clearly $\left(a_{n}\right) \notin c_{00}$, but $\left(a_{n}\right) \in \mathcal{I}$ hence $\left(a_{n}\right) \in A$. Note also that $\bigcup_{n=1}^{\infty} E_{n}=(0,1]$. Consider the function $f: \mathbb{R} \rightarrow \mathbb{R}$ defined by

$$
f(x)= \begin{cases}0 & \text { if } x \notin(0,1], \\ (-1)^{n} / \sqrt{n} & \text { if } x \in E_{n} .\end{cases}
$$

The transformed sequence $\left(f\left(a_{n}\right)\right) \in c s$ by Leibnitz's test for alternating series. However, $(f \cdot f)\left(a_{n}\right)=1 / n$ so $(f \cdot f) \notin c s$.

(iii) The proof is straightforward.

\section{Characterization Theorems}

In this section we characterize many of the spaces $F(A, B)$ in terms of wellknown and easily described classes of functions. Our results extend Theorem A, Theorem 2.6 and Theorem 2.10 in [1] and [2, Theorem 1]. In particular, the classes we consider include those of importance in [2, Theorem 1]: $f \in F(c s, c s)$ if and only if $f$ is linear near zero; and in [1, Theorem 2.10]: $f \in F\left(c s, l^{1}\right)$ if and only if $f$ is identically zero in a neighborhood of 0 . The classes of functions are as follows:

$$
\mathcal{F}=\{f: \mathbb{R} \rightarrow \mathbb{R}\}
$$

$B N Z$ (bounded near zero) $=\{f \in \mathcal{F}: \exists M, \delta>0$ such that $|f(x)|<$ $M \forall x \in(-\delta, \delta)\}$;

$C A Z$ (continuous at zero) $=\{f \in \mathcal{F}: f$ is continuous at 0$\}$;

$D A Z(g)$ (dominated at zero by $g)=\{f \in \mathcal{F}: \exists c, \delta>0$ such that $|f(x)| \leq$ $c|g(x)| \forall x \in(-\delta, \delta)\}$ (here $g \in \mathcal{F})$;

$L B$ (locally bounded) $=\left\{f \in \mathcal{F}: \forall n \in \mathbb{N}, \exists M_{n}>0\right.$ such that $|f(x)|<$ $\left.M_{n} \forall x \in(-n, n)\right\}$; 
$L N Z$ (linear near zero) $=\{f \in \mathcal{F}: \exists a \in \mathbb{R}, \delta>0$ such that $f(x)=$ ax $\forall x \in(-\delta, \delta)\}$;

$Z A Z$ (zero at zero) $=\{f \in \mathcal{F}: f(0)=0\}$

$Z E$ (zero everywhere) $=\{z\}$;

$Z N Z$ (zero near zero) $=\{f \in \mathcal{F}: \exists \delta>0$ such that $f(x)=0 \forall x \in$ $(-\delta, \delta)\}$.

Given a function $f \in \mathcal{F}$ we often write $f(\cdot)$ in place of the mapping notation $x \mapsto f(x)$. In particular, if $r \in(0, \infty)$ then $|\cdot|^{r}$ denotes the function $x \mapsto|x|^{r}$. The first result is a useful characterization of $F\left(l^{1}, l^{1}\right)$ and is essentially proved in [1, Theorem 2.6].

Proposition 3.1. $F\left(l^{1}, l^{1}\right)=D A Z(|\cdot|)$.

Proof. If $f \in F\left(l^{1}, l^{1}\right)$ then [1, Theorem 2.6] shows $f \in(Z A Z) \cap(C A Z)$. It follows from (5) in [1, Theorem 3.1] that $f \in D A Z(|\cdot|)$. Conversely, one also sees in (5) of [1, Theorem 3.1] that $f \in D A Z(|\cdot|)$ whenever $f \in F\left(l^{1}, l^{1}\right)$.

Proposition 3.1 is used next to give a similar characterization of $F\left(l^{p}, l^{q}\right)$ where $p, q \in(0, \infty)$.

Theorem 3.2. For $p, q \in(0, \infty)$ we have that $F\left(l^{p}, l^{q}\right)=D A Z\left(|\cdot|^{p / q}\right)$.

Proof. To prove $(\subseteq)$ let $f \in F\left(l^{p}, l^{q}\right)$. If $\left(a_{n}\right) \in l^{1}$ then $\left(\left|a_{n}\right|^{1 / p}\right) \in l^{p}$ and hence $\left(f\left(\left|a_{n}\right|^{1 / p}\right)\right) \in l^{q}$. It follows that the function $\left|f\left(\left.|\cdot|\right|^{1 / p}\right)\right|^{q} \in F\left(l^{1}, l^{1}\right)$ and similarly $\left|f\left(-|\cdot|^{1 / p}\right)\right|^{q} \in F\left(l^{1}, l^{1}\right)$. By Proposition 3.1 there exists $c, \delta>0$ such that $\left|f\left( \pm|x|^{1 / p}\right)\right|^{q} \leq c|x|$ for all $x \in(-\delta, \delta)$. Therefore, whenever $x \in$ $\left(-\delta^{1 / p}, \delta^{1 / p}\right)$,

$$
|f(x)| \leq|f(|x|)|+|f(-|x|)|=\left|f\left(\left.\left.|| x\right|^{p}\right|^{1 / p}\right)\right|+\left|f\left(-\left.\left.|| x\right|^{p}\right|^{1 / p}\right)\right| \leq 2 c^{1 / q}|x|^{p / q} .
$$

This proves $f \in D A Z\left(|\cdot|^{p / q}\right)$. For $(\supseteq)$ let $f \in D A Z\left(|\cdot|^{p / q}\right)$ and assume $c, \delta>0$ are such that $|f(x)| \leq c|x|^{p / q}$ for all $x \in(-\delta, \delta)$. If $\left(a_{n}\right) \in l^{p}$ then $\left|f\left(a_{n}\right)\right|^{q} \leq c^{q}\left|a_{n}\right|^{p}$ for all sufficiently large $n$. This implies $\left(f\left(a_{n}\right)\right) \in l^{q}$ by the comparison test and thus $f \in F\left(l^{p}, l^{q}\right)$.

Note in Proposition 3.1 and Theorem 3.2 that the characterization of $F\left(l^{p}, l^{q}\right)$ is expressed in terms of the ratio, $p / q$.

Corollary 3.3. Let $p, q \in(0, \infty)$. Then $F\left(l^{p t}, l^{q t}\right)=F\left(l^{p}, l^{q}\right)$ for each $t \in$ $(0, \infty)$.

Proof. The equality follows from the simple observation that $D A Z\left(|\cdot|^{p t / q t}\right)=$ $D A Z\left(|\cdot|^{p / q}\right)$ for each $t \in(0, \infty)$. 
Suppose $0<q<p<\infty$. It is easy to check using Theorem 3.2 that $f^{\prime}(0)=0$ for each $f \in F\left(l^{p}, l^{q}\right)$. However, the function $g \in \mathcal{F}$ defined by

$$
g(x)= \begin{cases}0 & \text { if } x \text { is irrational } \\ |x|^{p / q} & \text { if } x \text { is rational }\end{cases}
$$

shows there are elements of $F\left(l^{p}, l^{q}\right)$ for which zero is the only point of differentiability. When $0<p \leq q<\infty$ there are functions in $F\left(l^{p}, l^{q}\right)$ which are not differentiable at any point; take $g$ for example. In the special case when $p=q \in(0, \infty)$, Corollary 3.3 and [1, Theorem 2.6] imply that the four Dini derivatives of each function in $F\left(l^{p}, l^{q}\right)$ are finite. The four Dini derivatives can be distinct [1, Example 2.3].

Our next theorem is a characterization of $F(A, B)$ when $A$ or $B$ is $l^{\infty}$. The result extends (5) and shows that Theorem 3.2 is false if either $l^{p}$ or $l^{q}$ are replaced with $l^{\infty}$. Part (iii) shows that the inclusions in Proposition 2.3 become equalities when $q=\infty$.

Theorem 3.4. $\quad$ (i) $F\left(l^{\infty}, B\right)=Z E$ for all $B \in \mathcal{S} \backslash\left\{l^{\infty}\right\}$;

(ii) $F\left(c_{00}, l^{\infty}\right)=\mathcal{F}$;

(iii) $F\left(A, l^{\infty}\right)=B N Z$ for all $A \in \mathcal{S} \backslash\left\{c_{00}, l^{\infty}\right\}$;

(iv) $F\left(l^{\infty}, l^{\infty}\right)=L B$.

Proof. (i) To prove $(\subseteq)$, note from (1) and (2) that $c_{0}$ is the largest space in $\mathcal{S} \backslash\left\{l^{\infty}\right\}$. It suffices by Proposition 2.1(i) to prove the result for $c_{0}$. Let $f \in F\left(l^{\infty}, c_{0}\right)$ and $x \in \mathbb{R}$. The constant sequence $\left(a_{n}\right)$, where $a_{n}=x$, is an element of $l^{\infty}$ and hence $\left(f\left(a_{n}\right)\right) \in c_{0}$. This implies $f(x)=0$ for all $x \in \mathbb{R}$ which gives $(\subseteq)$. The inclusion $(\supseteq)$ is obvious.

(ii) The inclusion $(\subseteq)$ is obvious. For $(\supseteq)$ let $f \in \mathcal{F}$ and $\left(a_{n}\right) \in c_{00}$. For some natural number $m, a_{n} \neq 0$ for exactly $m-1$ values of $n$. So the transformed sequence $\left(f\left(a_{n}\right)\right)$ consists of at most $m$ distinct terms. This proves $f \in F\left(c_{00}, l^{\infty}\right)$.

(iii) To verify $(\subseteq)$ let $A \in \mathcal{S} \backslash\left\{c_{00}, l^{\infty}\right\}$ and $f \in \mathcal{F}$. If $f \notin B N Z$ then there exists a sequence $\left(a_{n}\right)$ with $\left|a_{n}\right| \in\left(0, n^{-n}\right)$ such that $\left|f\left(a_{n}\right)\right|>n$. The comparison test implies $\left(a_{n}\right) \in A$. However, $\left(f\left(a_{n}\right)\right) \notin l^{\infty}$ which is a contradiction. This proves $f \in B N Z$. For $(\supseteq)$ let $f \in B N Z$ and assume $M, \delta>0$ are such that $|f(x)|<M$ whenever $x \in(-\delta, \delta)$. If $\left(a_{n}\right) \in A$ then $a_{n} \rightarrow 0$ as $n \rightarrow \infty$. Therefore, $\left|a_{n}\right|<\delta$ for all sufficiently large $n$ which implies $\left|f\left(a_{n}\right)\right|<M$ for all but finitely many $n$. This shows $\left(f\left(a_{n}\right)\right) \in l^{\infty}$ which gives $(\supseteq)$. 
(iv) To establish $(\subseteq)$ let $f \in L B$. Assume for each natural $n$ there is a number $M_{n}>0$ such that $|f(x)|<M_{n}$ for all $x \in(-n, n)$. If $\left(a_{k}\right) \in l^{\infty}$ then $\left|a_{k}\right|<n_{0}$ for some $n_{0}$ so $\left|f\left(a_{k}\right)\right|<M_{n_{0}}$ for all $k$. This shows $\left(f\left(a_{k}\right)\right) \in l^{\infty}$ and thus $f \in F\left(l^{\infty}, l^{\infty}\right)$. For $(\supseteq)$ suppose $f \in \mathcal{F}$ but $f \notin L B$. Then for some fixed $n$ there exists $x_{k} \in(-n, n)$ such that $\left|f\left(x_{k}\right)\right|>k$ for each $k \in \mathbf{N}$. The sequence $\left(x_{k}\right) \in l^{\infty}$ but the transformed sequence $\left(f\left(x_{k}\right)\right) \notin l^{\infty}$ which is a contradiction.

When $A$ and $B$ are as in Theorem 3.4 we have the proper inclusions

$$
F\left(l^{\infty}, B\right) \subset F\left(l^{\infty}, l^{\infty}\right) \subset F\left(A, l^{\infty}\right) \subset F\left(c_{00}, l^{\infty}\right) .
$$

In part (iv) of Theorem 3.4, it is not necessary for a function to be bounded on $\mathbb{R}$ in order that it be an element of $F\left(l^{\infty}, l^{\infty}\right)$. Consider the simple example $f(x)=x$. Clearly $f \in L B$ and thus $f \in F\left(l^{\infty}, l^{\infty}\right)$.

We can now summarize conditions on the indices $p, q, r$ and $s$ which imply $F\left(l^{p}, l^{q}\right)$ is properly contained in $F\left(l^{r}, l^{s}\right)$.

Corollary 3.5. Let $(p, q),(r, s) \in(0, \infty] \times(0, \infty]$. Each of the following conditions imply the proper inclusion $F\left(l^{p}, l^{q}\right) \subset F\left(l^{r}, l^{s}\right)$ :

(i) $p=\infty$ and $q, r<\infty$;

(ii) $r<p<\infty$ and either $s=q<\infty$ or $s<q \leq \infty$;

(iii) $p<r<\infty, q<s$ and $q r<s p$;

(iv) $p=r<\infty$ and $q \leq s$.

Proof. (i) Combine parts (i) and (iii) of Theorem 3.4 with Theorem 3.2, and then observe that $Z E \subset D A Z\left(|\cdot|^{r / s}\right)$ when $s<\infty$, and that $Z E \subset B N Z$ when $s=\infty$.

(ii) When $s=q<\infty$ the assertion follows from Proposition 2.3(iii). When $s<q \leq \infty$, combine Proposition 2.3(i) with Proposition 2.3(iii) if $q<\infty$, and with Theorem 3.4(iii) if $q=\infty$.

(iii) For $t \in(0, \infty)$ the inequalities $p t>r$ and $q t<s$ are satisfied if $r / p<s / q$ which is equivalent to $q r<s p$. The assertion now follows from Corollary 3.3 and Proposition 2.1.

(iv) If $s<\infty$ then the result follows from Proposition 2.2(iii). If $s=\infty$ then combine Theorem 3.2 and Theorem 3.4(iii) and note that $D A Z\left(|\cdot|^{p / q}\right) \subset$ $B N Z$.

The next result deals with the spaces $F(A, B)$ when $A$ or $B$ is $c_{00}$ or $c_{0}$. 
Theorem 3.6. (i) $F\left(A, c_{00}\right)=Z N Z$ for all $A \in \mathcal{S} \backslash\left\{c_{00}, l^{\infty}\right\}$;

(ii) $F\left(c_{00}, B\right)=Z A Z$ for all $B \in \mathcal{S} \backslash\left\{l^{\infty}\right\}$;

(iii) $F\left(A, c_{0}\right)=(C A Z) \cap(Z A Z)$ for all $A \in \mathcal{S} \backslash\left\{c_{00}, l^{\infty}\right\}$;

(iv) $F\left(c_{0}, B\right)=Z N Z$ for all $B \in \mathcal{S} \backslash\left\{c_{0}, l^{\infty}\right\}$.

Proof. (i) To prove $(\subseteq)$, note from (1) that $\mathcal{I}$ is the smallest space in $\mathcal{S} \backslash\left\{c_{00}, l^{\infty}\right\}$. It is thus sufficient by Proposition 2.1(ii) to prove $F\left(\mathcal{I}, c_{00}\right) \subseteq$ $Z N Z$. Assume $f \in \mathcal{F}$ but $f \notin Z N Z$. Choose a strictly decreasing sequence $\left(a_{n}\right)$ such that $a_{n} \in\left(0, n^{-n}\right)$ and $f\left(a_{n}\right) \neq 0$. Then $\left(a_{n}\right) \in \mathcal{I}$ by the comparison test, but $\left(f\left(a_{n}\right)\right) \notin c_{00}$ which is a contradiction. To verify ( $\supseteq$ ) let $f \in Z N Z$ and assume $\delta>0$ is such that $f(x)=0$ for all $x \in(-\delta, \delta)$. If $\left(x_{n}\right) \in A$ then $x_{n} \in(-\delta, \delta)$ for all but finitely many $n$. Thus $f\left(x_{n}\right)=0$ for all but finitely many $n$ which shows $f \in F\left(A, c_{00}\right)$.

(ii) For $(\subseteq)$, it follows from Proposition 2.1(i) that we need only prove $F\left(c_{00}, c_{0}\right) \subseteq Z A Z$. If $f \in F\left(c_{00}, c_{0}\right)$ then $(f(0)) \in c_{0}$ since $(0) \in c_{00}$. This implies $f(0)=0$. To verify $(\supseteq)$ let $f \in Z A Z$ and $\left(a_{n}\right) \in c_{00}$. Since $a_{n}=0$ for all but finitely many $n, f\left(a_{n}\right)=0$ for all but finitely many $n$. This shows $f \in F\left(c_{00}, c_{00}\right)$ and from Proposition 2.1(i), $f \in F\left(c_{00}, B\right)$.

(iii) As in (i), to prove $(\subseteq)$ we need only verify that $F\left(\mathcal{I}, c_{0}\right) \subseteq(C A Z) \cap$ $(Z A Z)$. If $f \in F\left(\mathcal{I}, c_{0}\right)$ then $(f(0)) \in c_{0}$ since $(0) \in \mathcal{I}$. This implies $f(0)=$ 0 and hence $f \in Z A Z$. Suppose $f \notin C A Z$. There exists an $\epsilon>0$ and a sequence $\left(a_{n}\right)$ such that $\left|a_{n}\right| \in\left(0, n^{-n}\right)$ and $\left|f\left(a_{n}\right)\right|>\epsilon$. Then $\left(a_{n}\right) \in \mathcal{I}$ by the comparison test, but $\left(f\left(a_{n}\right)\right) \notin c_{0}$ which is a contradiction. This shows $f \in C A Z$. For $(\supseteq)$ let $f \in(C A Z) \cap(Z A Z)$. If $\left(a_{n}\right) \in c_{0}$ then $f\left(a_{n}\right) \rightarrow f(0)$ as $n \rightarrow \infty$ and $f(0)=0$. This proves the transformed sequence $\left(f\left(a_{n}\right)\right) \in c_{0}$ and hence $f \in F\left(A, c_{0}\right)$ by Proposition 2.1(ii).

(iv) To prove $(\subseteq)$ we first assume $B \subseteq c s$ and let $f \in F\left(c_{0}, B\right)$. It follows from Proposition 2.1 that $f \in F(c s, c s)$ and hence $f \in F^{(c p)}$ by (3). Following $[2$, Theorem 1] there exists $a \in \mathbb{R}$ and $\delta>0$ such that $f(x)=a x$ for all $x \in(-\delta, \delta)$. Choose a natural number $n_{0}>1 / \delta$. The sequence $\left(x_{n}\right)=\left(\left(n_{0}+\right.\right.$ $\left.n)^{-1}\right) \in c_{0}$ so $\left(f\left(x_{n}\right)\right) \in c s$. This implies $a=0$ and $f \in Z N Z$. Now assume $B \nsubseteq c s$. We establish $(\subseteq)$ by contradiction. Let $f \in F\left(c_{0}, B\right)$ and assume $f \notin$ $Z N Z$. Choose a sequence $\left(a_{n}\right) \in c_{0}$ such that $\left(f\left(a_{n}\right)\right) \notin c s$ and $\left|f\left(a_{n}\right)\right| \in(0,1]$. Let $m_{0}=0$ and for each natural $j$ let $m_{j} \in \mathbb{N}$ satisfy $m_{j}\left|f\left(a_{j}\right)\right|^{j} \geq 1 / j$. For each $k$ let $M_{k}=\sum_{j=0}^{k-1} m_{j}$. Define the sequence $\left(b_{n}\right)$ as follows: given $k$ and $i=1,2, \ldots, m_{k}$, let $b_{n}=a_{k}$ where $n=M_{k}+i$. The terms of $\left(b_{n}\right)$ agree with 
the terms of $\left(a_{n}\right)$ in succession on blocks of length $m_{k}$. This implies $\left(b_{n}\right) \in c_{0}$. Let $p, k \in \mathbb{N}$ with $k>p$. We have

$$
\sum_{n=1}^{M_{k+1}}\left|f\left(b_{n}\right)\right|^{p}=\sum_{j=1}^{k} m_{j}\left|f\left(a_{j}\right)\right|^{p} \geq \sum_{j=p}^{k} m_{j}\left|f\left(a_{j}\right)\right|^{j} \geq \sum_{j=p}^{k} 1 / j .
$$

It follows that the transformed sequence $\left(f\left(b_{n}\right)\right) \notin l^{p}$ so $f \notin \mathcal{U}$. Since $B \subseteq \mathcal{U}$, $f \notin F\left(c_{0}, B\right)$ which is a contradiction. This proves $f \in Z N Z$ which gives $(\subseteq)$. For $(\supseteq)$ let $f \in Z N Z$ and assume $\delta>0$ is such that $f(x)=0$ for all $x \in(-\delta, \delta)$. If $\left(a_{n}\right) \in c_{0}$ then $a_{n} \in(-\delta, \delta)$ for all but finitely many $n$ so $f\left(a_{n}\right)=0$ for all but finitely many $n$. This proves $f \in F\left(c_{0}, c_{00}\right)$ and hence $f \in B$ by Proposition 2.1(i).

In Theorem 3.6(iii), zero need be the only point of continuity of a function $f \in(C A Z) \cap(Z A Z)$. As an example, consider [1, Example 2.4] where $a \in$ $\mathbb{R}, a \neq 0$, and

$$
f(x)= \begin{cases}0 & \text { if } x \text { is irrational } \\ a x & \text { if } x \text { is rational. }\end{cases}
$$

We now characterize $F(A, B)$ where $A, B \in\{\mathcal{I}, \mathcal{U}\}$. Our results for these spaces are natural and appealing in the sense that $F(A, B)$ is expressible in terms of intersections and unions of $F\left(l^{p}, l^{q}\right)$-spaces.

Theorem 3.7. (i) $F(\mathcal{U}, \mathcal{I})=\bigcap_{0<p<\infty} \bigcap_{0<s<\infty} F\left(l^{p}, l^{s}\right)$;

(ii) $F(\mathcal{U}, \mathcal{U})=F(\mathcal{I}, \mathcal{U})=F(\mathcal{I}, \mathcal{I})=\bigcup_{0<p<\infty} \bigcup_{0<s<\infty} F\left(l^{p}, l^{s}\right)$.

Proof. (i) Since $F(\mathcal{U}, \mathcal{I}) \subseteq F\left(l^{p}, \mathcal{I}\right)$ for all $p \in(0, \infty)$, Proposition 2.2(ii) implies that

$$
F(\mathcal{U}, \mathcal{I}) \subseteq \bigcap_{0<p<\infty} F\left(l^{p}, \mathcal{I}\right)=\bigcap_{0<p<\infty} \bigcap_{0<s<\infty} F\left(l^{p}, l^{s}\right)
$$

For $(\supseteq)$ let $f \in \bigcap_{0<p<\infty} \bigcap_{0<s<\infty} F\left(l^{p}, l^{s}\right)$ and $\left(a_{n}\right) \in \mathcal{U}$. Then $\left(a_{n}\right) \in l^{r}$ for some $r \in(0, \infty)$ so the transformed sequence $\left(f\left(a_{n}\right)\right) \in \mathcal{I}$.

(ii) We first show that

$$
F(\mathcal{U}, \mathcal{U})=\bigcup_{0<p<\infty} \bigcup_{0<s<\infty} F\left(l^{p}, l^{s}\right)
$$


Since $F(\mathcal{U}, \mathcal{U}) \subseteq F\left(l^{p}, \mathcal{U}\right)$ for all $p \in(0, \infty)$, Proposition 2.2(i) implies that

$$
F(\mathcal{U}, \mathcal{U}) \subseteq \bigcup_{0<p<\infty} \bigcup_{0<s<\infty} F\left(l^{p}, l^{s}\right)
$$

Conversely, let $f \in \bigcup_{0<p<\infty} \bigcup_{0<s<\infty} F\left(l^{p}, l^{s}\right)$ so that $f \in F\left(l^{p}, l^{s}\right)$ for some $p, s \in(0, \infty)$. If $\left(a_{n}\right) \in \mathcal{U}$ then $\left(a_{n}\right) \in l^{r}$ for some $r \in(0, \infty)$, thus $f \in$ $F\left(l^{r}, l^{(r s) / p}\right)$ by Corollary 3.3. The transformed sequence $\left(f\left(a_{n}\right)\right) \in l^{(r s) / p}$ hence $\left(f\left(a_{n}\right)\right) \in \mathcal{U}$. It is obvious that $F(\mathcal{I}, \mathcal{I}) \subseteq F(\mathcal{I}, \mathcal{U})$. Suppose $f \in$ $\bigcup_{0<p<\infty} \bigcup_{0<s<\infty} F\left(l^{p}, l^{s}\right)$ and $\left(a_{n}\right) \in \mathcal{I}$. Let $q \in(0, \infty)$ and assume $p, s \in$ $(0, \infty)$ are such that $f \in F\left(l^{p}, l^{s}\right)$. Corollary 3.3 implies $f \in F\left(l^{(p q) / s}, l^{q}\right)$ thus $\left(f\left(a_{n}\right)\right) \in \mathcal{I}$. We now have that

$$
\bigcup_{0<p<\infty} \bigcup_{0<s<\infty} F\left(l^{p}, l^{s}\right) \subseteq F(\mathcal{I}, \mathcal{I}) \subseteq F(\mathcal{I}, \mathcal{U})
$$

We finish the proof by showing

$$
F(\mathcal{I}, \mathcal{U}) \subseteq \bigcup_{0<p<\infty} \bigcup_{0<s<\infty} F\left(l^{p}, l^{s}\right)
$$

The argument is by contradiction. Assume there is some $f \in F(\mathcal{I}, \mathcal{U})$ such that $f \notin F\left(l^{p}, l^{s}\right)$ for any $p, s \in(0, \infty)$. For each $k$ there exists a sequence $\left(a_{k, n}\right) \in l^{1 / k}$ such that $\left(f\left(a_{k, n}\right)\right) \notin l^{k}$. We can assume that

$$
\sum_{n=1}^{\infty}\left|a_{k, n}\right|^{1 / k} \leq 1 / 2^{k}
$$

and, by Theorem 3.6(iii), that $\left|f\left(a_{k, n}\right)\right| \leq 1$ for all $n$ and $k$. Let $m_{0}=0$ and define

$$
m_{k}=\min \left\{M \in \mathbb{N}: \sum_{n=1}^{M}\left|f\left(a_{k, n}\right)\right|^{k} \geq 1\right\} \text { and } M_{k}=\sum_{j=0}^{k-1} m_{j} .
$$

Let $\left(b_{m}\right)$ be the row-by-row enumeration of the set $\left\{a_{k, n}: k \in \mathbb{N}, n=\right.$ $\left.1,2, \ldots, m_{k}\right\}$. Therefore, given $k$ and $n=1,2, \ldots, m_{k}, m=M_{k}+n$. We show $\left(b_{m}\right) \in \mathcal{I}$ and $\left(f\left(b_{m}\right)\right) \notin \mathcal{U}$.

Let $r \in(0,1)$ and $k_{0}=\min \{k \in \mathbb{N}: k>1 / r\}$; note that $k_{0} \geq 2$. For all 
natural numbers $K>k_{0}$,

$$
\begin{aligned}
\sum_{m=1}^{M_{K+1}}\left|b_{m}\right|^{r} & =\sum_{k=1}^{K} \sum_{n=1}^{m_{k}}\left|a_{k, n}\right|^{r} \\
& \leq \sum_{k=1}^{k_{0}-1} \sum_{n=1}^{m_{k}}\left|a_{k, n}\right|^{r}+\sum_{k=k_{0}}^{K} \sum_{n=1}^{m_{k}}\left|a_{k, n}\right|^{1 / k} \\
& \leq c_{r}+\sum_{k=k_{0}}^{K} 1 / 2^{k} \\
& <c_{r}+1
\end{aligned}
$$

where $c_{r}$ is a constant which depends only on $r$. It follows that $\left(b_{m}\right) \in l^{r}$ which implies $\left(b_{m}\right) \in \mathcal{I}$.

Now let $r \in[1, \infty)$ and choose a natural number $k_{0}>r$. Then for each $K>k_{0}$,

$$
\sum_{m=1}^{M_{K+1}}\left|f\left(b_{m}\right)\right|^{r}=\sum_{k=1}^{K} \sum_{n=1}^{m_{k}}\left|f\left(a_{k, n}\right)\right|^{r} \geq \sum_{k=k_{0}}^{K} \sum_{n=1}^{m_{k}}\left|f\left(a_{k, n}\right)\right|^{k} \geq K-k_{0} .
$$

This shows that the transformed sequence $\left(f\left(b_{m}\right)\right) \notin l^{r}$ and implies $\left(f\left(b_{m}\right)\right) \notin$ $\mathcal{U}$. This is a contradiction.

In Theorem 3.7(iii) we have the equivalent expression

$$
\bigcup_{0<p<\infty} \bigcup_{0<s<\infty} F\left(l^{p}, l^{s}\right)=\bigcup_{0<r<\infty} D A Z\left(|\cdot|^{r}\right)
$$

which follows from Theorem 3.2 and Corollary 3.3. Furthermore, by Theorems 3.6 and 3.7 ,

$$
Z N Z \subseteq F(\mathcal{U}, \mathcal{I}) \subseteq F(\mathcal{U}, \mathcal{U}) \subseteq(C A Z) \cap(Z A Z)
$$

The following examples demonstrate that these inclusions are proper.

Example 3.8. For $i=1,2,3$, there are functions $f_{i} \in \mathcal{F}$ such that

(i) $f_{1} \in F(\mathcal{U}, \mathcal{I})$ and $f_{1} \notin Z N Z$;

(ii) $f_{2} \in F(\mathcal{U}, \mathcal{U})$ and $f_{2} \notin F(\mathcal{U}, \mathcal{I})$;

(iii) $f_{3} \in(C A Z) \cap(Z N Z)$ and $f_{3} \notin F(\mathcal{U}, \mathcal{U})$. 
Proof. (i) If $E_{n}=\left((n+1)^{-1}, n^{-1}\right]$ then $(0,1]=\bigcup_{n=1}^{\infty} E_{n}$ and the union is disjoint. Let $f_{1}$ be defined by

$$
f_{1}(x)= \begin{cases}0 & \text { if } x \notin(0,1] \\ |x|^{n} & \text { if } x \in E_{n}\end{cases}
$$

Clearly $f_{1} \notin Z N Z$. Given $p, s \in(0, \infty)$, choose a natural number $n_{0} \geq p / s$ and let $x \in\left(0, n_{0}\right)$. Since $x \in E_{n}$ for some $n>n_{0}$,

$$
f_{1}(x)=|x|^{n}<|x|^{n_{0}} \leq|x|^{p / s} .
$$

This implies $f_{1} \in D A Z\left(|\cdot|^{p / s}\right)$ for all $p, s \in(0, \infty)$ and therefore $f_{1} \in F(\mathcal{U}, \mathcal{I})$ by Theorem 3.7(i) and Theorem 3.2.

(ii) From Theorem 3.7 we can take the simple example $f_{2}(x)=|x|$.

(iii) Consider the function $f_{3}$ defined by

$$
f_{3}(x)= \begin{cases}0 & \text { if } x \notin(0,1), \\ (\log (x))^{-1} & \text { if } x \in(0,1) .\end{cases}
$$

It is obvious that $f_{3} \in Z A Z$ and it is easy to check that $f_{3} \in C A Z$. To show that $f_{3} \notin F(\mathcal{U}, \mathcal{U})$, it is sufficient by Theorem 3.7(ii) and Theorem 3.2 to verify $f_{3} \notin D A Z\left(|\cdot|^{1 / k}\right)$ for any $k \in \mathbb{N}$. By l'Hopital's rule we see that $\log (x) x^{1 / k} \rightarrow 0$ as $x \rightarrow 0^{+}$. This implies there does not exist $c, \delta>0$ such that $\left|f_{3}(x)\right| \leq c|x|^{1 / k}$ for all $x \in(-\delta, \delta)$. Therefore $f_{3} \notin D A Z\left(|\cdot|^{1 / k}\right)$ so $f \notin F(\mathcal{U}, \mathcal{U})$.

In the final theorem we characterize the spaces $F(A, B)$ where $A$ or $B$ is $c s$. Some of these characterizations have already been established in previous theorems or are known results in the literature. Most notably, recall the following results which motivated our present investigation: $F(c s, c s)=L N Z$, [2, Theorem 1]; $F\left(c s, l^{1}\right)=Z N Z$, [1, Theorem 2.10]; $F\left(l^{1}, c s\right)=D N Z(|\cdot|)$, [1, Theorem 2.6]. Furthermore, Theorems 3.4 and 3.6 give characterizations of $F(c s, B)$ where $B \in\left\{c_{0}, l^{\infty}\right\}$ and of $F(A, c s)$ where $A \in\left\{c_{00}, c_{0}, l^{\infty}\right\}$. The constructions in the next theorem are similar, but slightly more intricate, to those in Proposition 2.2(i), Theorem 3.6(iv) and Theorem 3.7(ii). We give two proofs of part (iii) below. The first is a simple consequence of Theorem 3.7(iii). The second proof involves a construction which is interesting in its own right and does not depend on previous results.

Theorem 3.9. (i) $F(c s, \mathcal{U})=Z N Z$;

(ii) $F\left(l^{p}, c s\right)=D A Z\left(|\cdot|^{p}\right)$ for all $p \in(0, \infty)$;

(iii) $F(\mathcal{I}, c s)=\bigcup_{0<p<\infty} F\left(l^{p}, c s\right)$; 
(iv) $F(\mathcal{U}, c s)=\bigcap_{0<p<\infty} F\left(l^{p}, c s\right)$.

Proof. (i) It is easy to check $(\supseteq)$ either directly from Definition 1.1 or from Theorem 3.6(iv) and Proposition 2.1(ii). To prove $(\subseteq)$ we show first that $F\left(c s, l^{q}\right) \subseteq Z N Z$ for all $q \in(0, \infty)$. By [1, Theorem 2.10] and (1) it is sufficient to take $q \in(1, \infty)$. Let $f \in F\left(c s, l^{q}\right)$ and $\left(a_{n}\right) \in c s$. The function $|f(\cdot)|^{q} \in F\left(c s, l^{1}\right)$ and [1, Theorem 2.10] implies $|f(\cdot)|^{q} \in Z N Z$. Therefore, $f \in Z N Z$ so we now have

$$
Z N Z=\bigcup_{0<q<\infty} F\left(c s, l^{q}\right) \subseteq F(c s, \mathcal{U}) .
$$

By way of contradiction assume there is some $f \in F(c s, \mathcal{U})$ such that $f \notin$ $Z N Z$. For each natural $k$ there exists a sequence $\left(a_{k, n}\right) \in c s$ such that the transformed sequence $\left(f\left(a_{k, n}\right)\right) \notin l^{k}$. By the Cauchy criterion there exists $c_{k} \in \mathbb{N}$ such that $r_{2} \geq r_{1} \geq c_{k}$ implies $\left|\sum_{n=r_{1}}^{r_{2}} a_{k, n}\right| \leq 1 / 2^{k}$. Define

$$
d_{k}=\min \left\{d \in \mathbb{N}: \sum_{n=c_{k}}^{d}\left|f\left(a_{k, n}\right)\right|^{k} \geq 1\right\} .
$$

Let $m_{0}=0, m_{k}=d_{k}-c_{k}+1$ and $M_{k}=\sum_{j=0}^{k-1} m_{j}$. Let $\left(b_{m}\right)$ be the row-by-row enumeration of the set $\left\{a_{k, n}: k \in \mathbb{N}, n=c_{k}, \ldots, d_{k}\right\}$ such that the $k^{\text {th }}$ block of length $m_{k}$ is enumerated completely before the $(k+1)^{s t}$ block. Thus for each $k$ and $n=c_{k}, \ldots, d_{k}, m=M_{k}+n$. We show $\left(b_{m}\right) \in c s$ and $\left(f\left(b_{m}\right)\right) \notin \mathcal{U}$.

Let $\epsilon>0$ and choose $K$ such that $\sum_{k=K}^{\infty} 1 / 2^{k}<\epsilon$. Given $r_{1}, r_{2} \in \mathbb{N}$ with $r_{2} \geq r_{1} \geq M_{K}+1$, define

$$
k_{i}=\min \left\{k \in \mathbb{N}: M_{k}<r_{i} \leq M_{k+1}\right\}, i=1,2 .
$$

Since $k_{2} \geq k_{1} \geq K$, it follows from the triangle inequality that

$$
\left|\sum_{m=r_{1}}^{r_{2}} b_{m}\right| \leq \sum_{k=k_{1}}^{k_{2}} 1 / 2^{k} \leq \sum_{k=K}^{\infty} 1 / 2^{k}<\epsilon .
$$

The Cauchy criterion now implies $\left(b_{m}\right) \in c s$.

To see that $\left(f\left(b_{m}\right)\right) \notin \mathcal{U}$, refer to the proof of Theorem 3.7(iii). This produces the contradiction.

(ii) The proof of $(\subseteq)$ is similar to the proof of $(\subseteq)$ in Theorem 3.2 except that we replace $l^{q}$ with $c s$ and replace $D A Z\left(|\cdot|^{p / q}\right)$ with $D A Z\left(|\cdot|^{p}\right)$. The 
details are left to the reader. For $(\supseteq)$ replace $l^{q}$ with $c s$ and see the proof of $(\supseteq)$ in Theorem 3.2 .

(iii) (Proof 1.) Since $F(\mathcal{I}, \mathcal{I}) \subseteq F(\mathcal{I}, c s) \subseteq F(\mathcal{I}, \mathcal{U})$, the result follows trivially from Theorem 3.7(iii) and (6).

(Proof 2.) For $\left(\subseteq\right.$ ) note from (ii) that $D A Z\left(|\cdot|^{p}\right) \subseteq F(\mathcal{I}, c s)$ for all $p \in$ $(0, \infty)$. Therefore,

$$
\bigcup_{0<p<\infty} D A Z\left(|\cdot|^{p}\right) \subseteq F(\mathcal{I}, c s) .
$$

We prove $(\supseteq)$ by contradiction. Assume there exists $f \in F(\mathcal{I}, c s)$ such that $f \notin F\left(l^{p}, c s\right)$ for any $p \in(0, \infty)$. For each $k$ there is a sequence $\left(a_{k, n}\right) \in l^{1 / k}$ such that the transformed sequence $\left(f\left(a_{k, n}\right)\right) \notin c s$. As in part (ii) above and in Theorem 3.7(ii), we construct a subsequence $\left(b_{m}\right)$ of the infinite array $\left\{a_{k, m}\right.$ : $k, n \in \mathbb{N}\}$ satisfying $\left(b_{m}\right) \in \mathcal{I}$ and $\left(f\left(b_{m}\right)\right) \notin c s$.

Use the Cauchy criterion to select $\epsilon_{k}>0$ with the property that for each $N \in \mathbb{N},\left|\sum_{n=c}^{d} f\left(a_{k, n}\right)\right| \geq \epsilon_{k}$ whenever $c \geq d \geq N$. Define $s_{k}=\min \{s \in$ $\left.\mathbb{N}: s \geq 1 / \epsilon_{k}\right\}$ and note $\epsilon_{k} s_{k} \geq 1$. Choose a natural number $N_{k}$ such that $\sum_{n=N_{k}}^{\infty}\left|a_{k, n}\right|^{1 / k} \leq \frac{1}{2^{k} s_{k}}$. Now select natural numbers $d_{k} \geq c_{k} \geq N_{k}$ which satisfy $\sum_{n=c_{k}}^{d_{k}}\left|a_{k, n}\right|^{1 / k} \leq \frac{1}{2^{k} s_{k}}$ and $\left|\sum_{n=c_{k}}^{d_{k}} f\left(a_{k, n}\right)\right| \geq \epsilon_{k}$ Let $m_{0}=s_{0}=$ $0, m_{k}=d_{k}-c_{k}+1$ and let $M_{k}=\sum_{j=0}^{k-1} m_{j} s_{j}$. Define the sequence $\left(b_{m}\right)$ such that for each $k, b_{m}$ replicates $s_{k}$ copies of the $k^{t h}$ block, $a_{k, c_{k}}, \ldots, a_{k, d_{k}}$. Thus for each $k$ and $n=1,2, \ldots, s_{k} m_{k}, m=M_{k}+n$.

To prove $\left(b_{m}\right) \in \mathcal{I}$ let $r \in(0,1)$. Let $k_{0}=\min \{k \in \mathbb{N}: k>1 / r\}$ and note $k_{0} \geq 2$. For each natural $K>k_{0}$,

$$
\begin{aligned}
\sum_{m=1}^{M_{K+1}}\left|b_{m}\right|^{r} & =\sum_{k=1}^{K} s_{k}\left(\sum_{n=c_{k}}^{d_{k}}\left|a_{k, n}\right|^{r}\right) \\
& \leq \sum_{k=1}^{k_{0}-1} s_{k}\left(\sum_{n=c_{k}}^{d_{k}}\left|a_{k, n}\right|^{r}\right)+\sum_{k=k_{0}}^{K} s_{k}\left(\sum_{n=c_{k}}^{d_{k}}\left|a_{k, n}\right|^{1 / k}\right) \\
& \leq c_{r}+\sum_{k=k_{0}}^{K} 1 / 2^{k} \\
& <c_{r}+1
\end{aligned}
$$

where $c_{r}$ is a constant which depends only on $r$. This proves $\left(b_{m}\right) \in l^{r}$ and implies $\left(b_{m}\right) \in \mathcal{I}$.

To show that the transformed sequence $\left(f\left(b_{m}\right)\right) \notin c s$ let $\epsilon>0$ and $M \in \mathbb{N}$. If we choose $k$ such that $M_{k}+1 \geq M$, then $M_{k+1} \geq M_{k}+1 \geq M$. Since the 
numbers $s_{k}>0$, we have that

$$
\left|\sum_{m=M_{k}+1}^{M_{k+1}} f\left(b_{m}\right)\right|=\left|s_{k} \sum_{n=c_{k}}^{d_{k}} f\left(a_{k, n}\right)\right| \geq s_{k} \epsilon_{k} \geq 1 .
$$

Therefore, the Cauchy criterion fails for the sequence $\left(f\left(b_{m}\right)\right)$ so $\left(f\left(b_{m}\right)\right) \notin c s$. This is a contradiction which ends the proof of (iii).

(iv) For $(\subseteq)$ we have that $F(\mathcal{U}, c s) \subseteq F\left(l^{p}, c s\right)$ for all $p \in(0, \infty)$, thus

$$
F(\mathcal{U}, c s) \subseteq \bigcap_{0<p<\infty} F\left(l^{p}, c s\right) .
$$

To verify () let $f \in \bigcap_{0<p<\infty} F\left(l^{p}, c s\right)$ and $\left(a_{n}\right) \in \mathcal{U}$. Then $\left(a_{n}\right) \in l^{p}$ for some $p \in(0, \infty)$ which implies $\left(f\left(a_{n}\right)\right) \in c s$.

Example 3.8 and Theorem 3.9 establish the proper inclusions

$$
Z N Z \subset F(\mathcal{U}, c s) \subset F(\mathcal{I}, c s) \subset(C A Z) \cap(Z A Z) .
$$

Corollary 3.10. $\quad$ (i) $F(\mathcal{U}, \mathcal{I})=F(\mathcal{U}, c s)$;

(ii) $F(\mathcal{U}, \mathcal{U})=F(\mathcal{I}, c s)$.

Proof. (i) Proposition 2.1(i) implies $(\subseteq)$. To check $(\supseteq)$ let $p, s \in(0, \infty)$ and let $r=p / s$. By Theorem 3.2 and Corollary 3.3 we have

$$
D A Z\left(|\cdot|^{r}\right)=F\left(l^{r}, l^{1}\right)=F\left(l^{p / s}, l^{1}\right)=F\left(l^{p}, l^{s}\right) .
$$

The result now follows from Theorem 3.9(iv) and Theorem 3.7(i).

(ii) This is obvious from Theorem 3.7(ii), Theorem 3.9(iii) and (6).

\section{Acknowledgments}

The author acknowledges the helpful discussions with Professors Marshall Ash, Krzysztof Ciesielski and Paul Humke during the Summer Symposium in Real Analysis, XXII, at Santa Barbara. Their suggestions regarding the construction of several sequences is appreciated.

\section{References}

[1] J. Borsík, J. Červeňanský and T. Šalát, Remarks on functions preserving convergence of infinite series, Real Analysis Exchange, 21 (1995-96), 725731. 
[2] R. Rado, A theorem on infinite series, J. Lond. Math. Soc., 35 (1960), $273-276$.

[3] W. Ruckle, Sequence spaces, Pitman Research Notes in Mathematics, 49, London, 1981.

[4] A. Smith, Convergence preserving functions, Amer. Math. Monthly, 98 (1991), 831-833.

[5] K. Stromberg, An introduction to classical real analysis, Wadsworth International, Belmont, 1981.

[6] G. Wildenberg, Convergence preserving functions, Amer. Math. Monthly, 95 (1988), 542-544. 